\title{
EL USO DE LA ENCUESTA DE TIPO SOCIAL EN TRADUCTOLOGÍA. CARACTERÍSTICAS METODOLÓGICAS
}

\author{
Anna Kuznik, Amparo Hurtado Albir, \\ Anna Espinal Berenguer \\ Universitat Autònoma de Barcelona
}

\section{Resumen}

La traducción es una actividad desempeñada por un colectivo laboral y profesional (los traductores), en algunos casos con un proceso formativo previo, a cargo de empleadores, y dirigida a usuarios de la traducción. Estos cuatro colectivos (traductores, alumnos de traducción, empleadores y usuarios), con características sociales bien definidas, son colectivos susceptibles de ser estudiados con uno de los métodos de investigación más frecuentes en las ciencias sociales: la encuesta.

En el presente artículo se dan a conocer las características metodológicas de la encuesta de tipo social. Se ejemplifica y valora su aplicación en la Traductología en diversos tipos de estudios: estudios orientados a preparar reformas universitarias, a detectar competencias profesionales y a recoger opiniones en torno a la calidad de la interpretación y subtitulación.

\begin{abstract}
Translation is an activity carried out by professionals - in some cases after a period of formal training - who are employed or self-employed, and whose work is destined for translation users. Translators, translator trainees, employers of translators, and translation users are four clearly defined social groups within the translation industry that may be the subject of study using one of the methods most frequently used within the field of social sciences: the social survey.

This paper presents the characteristics of social survey methodology. The use of social surveys in the field of Translation Studies when preparing to introduce changes in academic curricula, defining professional competencies, or obtaining opinions regarding the quality of interpreting and subtitling is analysed and evaluated.
\end{abstract}




\section{Palabras clave}

Encuesta. Método de investigación social. Colectivo. Traductología. Aspectos laborales y profesionales de la traducción/interpretación.

\section{Keywords}

Survey. Social research methods. Population. Translation Studies. Work-related and professional aspects of translation and interpreting. 


\section{La encuesta como método de investigación social}

Desde tiempos ancestrales el ser humano ha recogido informaciones de toda índole sobre las actitudes, opiniones, costumbres e incidencias puntuales en un territorio dado. Hoy en día, la encuesta se ha convertido en una herramienta fundamental para el estudio de las relaciones sociales. Las organizaciones contemporáneas políticas, económicas y sociales, utilizan esta técnica como un instrumento indispensable para conocer el comportamiento de sus grupos de interés y tomar decisiones sobre ellos. De todas las técnicas de análisis social, la encuesta es la más representativa, debido a su intenso uso y difusión.

\subsection{Definición y características metodológicas de la encuesta}

La encuesta es una técnica de recogida de datos, o sea una forma concreta, particular y práctica de un procedimiento de investigación. Se enmarca en los diseños no experimentales de investigación empírica propios de la estrategia cuantitativa, ya que permite estructurar y cuantificar los datos encontrados y generalizar los resultados a toda la población estudiada. Permite recoger datos según un protocolo establecido, seleccionando la información de interés, procedente de la realidad, mediante preguntas en forma de cuestionario (su instrumento de recogida de datos). Se trata de un tipo de investigación interdisciplinario por excelencia, debido a su amplitud, a los requisitos que tiene que cumplir toda investigación de campo y al análisis estadístico de datos.

Si cotejamos la encuesta con las otras tres técnicas de obtención de datos (entrevista, grupo de discusión y observación directa), es la más adecuada para los estudios que tienen por objetivo recoger información extensiva de grandes poblaciones, donde se busca la representatividad estadística y el tratamiento matemático de datos. Entre sus ventajas cabe destacar la simplificación de la realidad, la gran posibilidad de tratamiento de datos y la representatividad conocida. Sus mayores limitaciones consisten en proporcionar una imagen simplificada y superficial de la realidad, basada en datos atomizados y descontextualizados, que no permiten tener una actitud comprensiva frente a los fenómenos estudiados. Desde el punto de vista práctico, es la técnica de recogida de datos más frágil, ya que siendo larga y costosa, puede fallar en 
muchas etapas del proceso. Esta desventaja se ve agravada por el hecho de que una vez se ha empezado a aplicar, no permite ninguna rectificación durante el proceso de recogida de datos; de ahí proviene su gran rigidez (Cea D'Ancona 1996, Valles 1997, Quivy y Campenhoudt 2005).

El carácter no experimental de la encuesta se basa en el hecho de que no se define ninguna variable independiente, sino que se pretende recoger información exclusivamente sobre las variables dependientes. Dichas variables dependientes corresponden a los aspectos y temas de interés que se pretenden estudiar, y que se definen en el marco teórico de cada encuesta. Las variables dependientes (factores o parámetros de estudio), tras la operativización, se convierten en las preguntas de los cuestionarios.

En este sentido, resulta imprescindible prestar mucha atención a los dos elementos principales de la encuesta: el diseño de la muestra y la elaboración del cuestionario. La muestra y el cuestionario son dos pilares fundamentales en los que se apoya cada encuesta. Esta condición queda reflejada en algunas definiciones de la encuesta que se han propuesto hasta la actualidad. Es el caso de la propuesta de León y Montero, quienes la definen como "una investigación destinada a conocer características de una población de sujetos a través de un conjunto de preguntas" (León y Montero 1993: 98). En la misma línea se sitúa la definición de Arnau, para quien la encuesta abarca:

un conjunto de técnicas e instrumentos de recopilación de datos que permite registrar o medir una gran cantidad de variables dependientes sin la manipulación activa de las condiciones de producción de los fenómenos o variables independientes. (Arnau 1995: 41)

La encuesta, además de ser una técnica de recogida de datos, se ha convertido en un método muy usual de investigación social, dada su gran versatilidad, la variedad de campos de aplicación, así como su capacidad de describir las características sociales de los colectivos estudiados e inferir conclusiones extensivas a la totalidad de dichos colectivos.

Existen muchos tipos de encuestas; López Romo (1998) propone una tipología basada en diez criterios. Partiendo de dicha propuesta planteamos la siguiente clasificación de la encuesta agrupando estos diez criterios en tres bloques: aspectos relacionados con el diseño general del estudio, con la población y la muestra, y con el instrumento de recogida de datos (Tabla 1). 


\begin{tabular}{|c|c|}
\hline \multicolumn{2}{|r|}{ Según el diseño general del estudio } \\
\hline $\begin{array}{l}\text { Área de interés de } \\
\text { los resultados } \\
\text { (o campo de } \\
\text { aplicación) }\end{array}$ & $\begin{array}{l}\text { - Mercadotecnia } \\
\text { - Medios de comunicación y publicidad } \\
\text { - Opinión pública (comportamiento político del ciudadano) } \\
\text { - Salud } \\
\text { - Cultura y sociedad (estudios académicos) } \\
\text { - Organizaciones (comportamiento de las entidades morales o } \\
\text { encuestas business to business) } \\
\text { - Demografía }\end{array}$ \\
\hline Temas abordados & $\begin{array}{l}\text { - Valores } \\
\text { - Necesidades, gustos e intereses } \\
\text { - Conocimientos } \\
\text { - Actitudes e imágenes } \\
\text { - Opiniones } \\
\text { - Intenciones } \\
\text { - Conductas, usos, hábitos } \\
\text { - Datos demográficos }\end{array}$ \\
\hline $\begin{array}{l}\text { Propósito } \\
\text { (objetivos } \\
\text { perseguidos) }\end{array}$ & $\begin{array}{l}\text { - Encuesta estratégica, de carácter exploratorio. Información am- } \\
\text { plia para diseñar y planear una actividad } \\
\text { - Encuesta táctica, de carácter concluyente. Información específica } \\
\text { para el desarrollo (implementación) de proyectos o evaluación y } \\
\text { seguimiento (monitoreo) de proyectos desarrollados }\end{array}$ \\
\hline Periodicidad & $\begin{array}{l}\text { - Encuesta unitaria (única en el tiempo) } \\
\text { - Encuesta de seguimiento (temas explorados a través del tiempo). } \\
\text { Muestras independientes cada vez (diseño transversal) o la misma } \\
\text { muestra durante todo el tiempo (diseño longitudinal o de panel) }\end{array}$ \\
\hline $\begin{array}{l}\text { Enfoque } \\
\text { metodológico } \\
\text { (nivel de } \\
\text { conocimiento que } \\
\text { pretende alcanzar } \\
\text { una encuesta) }\end{array}$ & $\begin{array}{l}\text { - Encuesta exploratoria. Objetivos: tener un primer acercamiento } \\
\text { al fenómeno o tema estudiado, identificar características generales } \\
\text { del problema, establecer hipótesis de trabajo, identificar las catego- } \\
\text { rías de análisis o el esquema conceptual, proporcionar ideas sobre } \\
\text { el diseño de muestras y cálculos estadísticos. Se estudian pocos } \\
\text { casos; muestra representativa o no representativa (muestra de cri- } \\
\text { terio); preguntas abiertas. } \\
\text { - Encuesta descriptiva. Objetivos: describir con precisión -normal- } \\
\text { mente con porcentajes y promedios- las características del fenó- } \\
\text { meno observado; como consecuencia de dicha descripción, iden- } \\
\text { tificar y cuantificar con precisión la relevancia de cada uno de los } \\
\text { aspectos estudiados en la etapa exploratoria que puede o no estar } \\
\text { relacionada con una hipótesis de trabajo, determinar los aspectos } \\
\text { relevantes. Muestra representativa. } \\
\text { - Encuesta explicativa (evaluativa). Objetivos: describir la frecuen- } \\
\text { cia con que ocurre un fenómeno asociado a otro, señalar relaciones } \\
\text { entre factores que constituyen un fenómeno, sin establecer causa- } \\
\text { lidad. Muestra representativa. } \\
\text { - Encuesta causal. Objetivos: marcar una relación causal entre las } \\
\text { variables. Encuesta combinada con diseño experimental o cuasi } \\
\text { experimental. En sentido estricto: sólo los diseños experimentales } \\
\text { permiten establecer esta relación de causalidad entre las variables. } \\
\text { Muestra rigurosamente seleccionada. }\end{array}$ \\
\hline
\end{tabular}




\begin{tabular}{|c|c|}
\hline $\begin{array}{l}\text { Destino de la } \\
\text { información } \\
\text { (patrocinio) }\end{array}$ & $\begin{array}{l}\text { - Encuesta ad hoc (un patrocinador o cliente único solicita y cubre } \\
\text { en su totalidad los costos del estudio) } \\
\text { - Encuestas de multicliente o sindicadas (ofrecen información de } \\
\text { interés amplio; resultados compartidos por varios usuarios, los } \\
\text { cuales sufragan los gastos de manera compartida) } \\
\text { - Encuesta de difusión pública (fines académicos; el propósito es el } \\
\text { conocimiento en sí mismo) }\end{array}$ \\
\hline \multicolumn{2}{|r|}{ Según la población y la muestra } \\
\hline $\begin{array}{l}\text { Unidad estudiada } \\
\text { (unidad de análisis) }\end{array}$ & $\begin{array}{l}\text { - Consumidores o usuarios (mercadotecnia) } \\
\text { - Audiencia (medios de comunicación) } \\
\text { - Ciudadanos (estudios de opinión pública) } \\
\text { - Población (encuestas demográficas, culturales y sociales estudian } \\
\text { personas como integrantes de grupos) } \\
\text { - Entidades comerciales (mercadotecnia estudia establecimientos } \\
\text { comerciales que distribuye los productos o servicios) } \\
\text { - Organizaciones (encuestas de la mercadotecnia industrial o para } \\
\text { la comprensión del comportamiento macrosocial estudian las ins- } \\
\text { tituciones económicas, políticas o sociales; la unidad de análisis } \\
\text { es la organización pero se selecciona un informante calificado que } \\
\text { represente el interés y el punto de vista de la entidad) }\end{array}$ \\
\hline $\begin{array}{l}\text { Tipo de muestreo } \\
\text { (desde el punto de } \\
\text { vista estadístico) }\end{array}$ & $\begin{array}{l}\text { - Muestreo probabilístico (muestra representativa de la población) } \\
\text { - Muestreo no probabilístico (encuesta exploratoria) }\end{array}$ \\
\hline \multicolumn{2}{|r|}{ Según el instrumento de recogida de datos (cuestionario) } \\
\hline $\begin{array}{l}\text { Forma de registrar } \\
\text { la información }\end{array}$ & $\begin{array}{l}\text { - Cuestionario de tipo papel y lápiz (encuestador o de manera } \\
\text { autoaplicada) } \\
\text { - Ayudas automatizadas (CATI - Computer Assisted Telephone } \\
\text { Interview; CAPI - Computer Assisted Personal Interwiev) }\end{array}$ \\
\hline $\begin{array}{l}\text { Tipo de } \\
\text { administración }\end{array}$ & $\begin{array}{l}\text { - Administración personal } \\
\text { - Administración telefónica } \\
\text { - Por correo tradicional } \\
\text { - Por correo electrónico }\end{array}$ \\
\hline
\end{tabular}

Tabla 1. Tipología de encuestas (basado en López Romo 1998)

La encuesta ofrece todas las posibilidades de triangulación mencionadas por Denzin (1970, cit. en Flick 2004: 178 y en Hurtado Albir y Alves 2009: 70), quien enfoca la triangulación como una estrategia de validación mutua e identifica cuatro formas básicas:

1. Triangulación de fuentes de datos. Se combinan datos obtenidos de fuentes distintas: en momentos diferentes (tiempo), en sitios diferentes (espacio) o de distintos informantes (personas).

2. Triangulación de investigadores. Se combinan datos obtenidos por investigadores diferentes para evitar el sesgo del subjetivismo de cada uno. 
3. Triangulación de teorías. Se combinan diferentes teorías, hipótesis o puntos de vista teóricos sobre el mismo conjunto de datos. De esta manera se puede valorar la fuerza explicativa del fenómeno observado por teorías diferentes.

4. Triangulación metodológica. Es el concepto fundamental de Denzin, que distingue una triangulación "intra-método", o sea dentro del mismo método, y entre métodos distintos, combinando la estrategia cualitativa y cuantitativa. ${ }^{1}$ Esta última forma de triangulación (entre métodos distintos) sigue siendo la manera más común de entender la triangulación metodológica. De esta manera también se entiende la triangulación en el ámbito de Traductología, como "una perspectiva multimetodológica que permite explicar un fenómeno desde varios puntos de vista, combinando métodos cuantitativos y cualitativos" (Munday 2009: 237; entrada del glosario Hurtado Albir y Alves).

La encuesta es un método de investigación caro, lento y laborioso. Todas las operaciones implicadas en su ejecución representan un coste muy elevado: el diseño y la recogida de datos para una base de datos fiable; el diseño y la creación o la compra de las bases de datos de la población elegida; su mantenimiento y actualización. También suele ser laborioso y costoso el procedimiento de envío, recepción de respuestas, lectura de datos, análisis y elaboración del informe final.

\subsection{Estudio de colectivos: muestra y muestreo}

Hemos señalado anteriormente que la selección de la muestra constituye uno de los dos pilares básicos de la técnica de encuesta (aparte del diseño del cuestionario). Para referirse al conjunto de elementos que interesa estudiar mediante la encuesta, distintos autores usan el concepto de universo, población o colectivo. Ander-Egg menciona que se puede distinguir entre universo (hipotético) y población: "con el primer término se designa un conjunto de elementos, seres u objetos, y con el segundo un conjunto de números obtenidos midiendo y contando ciertas características de los mismos" (1990: 179). El concepto de universo tendría, pues, un carácter más hipotético, abstracto, indefinido; y el de población, más concreto en términos de definición numérica de las características de interés, propias de la población estudiada.

1. Los investigadores cada vez subrayan más la importancia de tratar la estrategia cuantitativa y cualitativa como planteamientos complementarios y de proporcionar diseños de investigación multimétodo (métodos mixtos) en los cuales los métodos cualitativos se integren con los cuantitativos (Bericat 1998, Creswell 2003, Verd y López 2008). 
Para realizar una investigación, no necesariamente hay que estudiar la totalidad de un colectivo, basta con elegir una muestra representativa del mismo. Esta muestra es elegida por determinados procedimientos (técnicas de muestreo) y su estudio conduce a conclusiones que son extensivas a la totalidad de la población, con una significativa economía de coste, mayor precisión y rapidez de ejecución.

Las técnicas de muestreo se apoyan en un censo previo del colectivo, en un listado que incluya todos los elementos de la población estudiada (censo, padrón electoral, catálogo, lista, mapa, plano). Ander-Egg lo denomina "base de la muestra" ("marco muestral" para López Romo 1998) y contempla también casos en los cuales no existe tal base muestral previa y tiene que ser constituida ad hoc, ya que no todos los colectivos están censados o catalogados.

Según el investigador opte por usar estrategias cualitativas o cuantitativas en la investigación, aplicará uno u otro criterio para asegurarse que su muestra es representativa para el colectivo. En el caso de estrategias cualitativas, el criterio suele ser teórico; para las estrategias cuantitativas -el caso de la encuesta- el criterio suele ser estadístico, probabilístico.

Las muestras no probabilísticas, seleccionadas con criterio teórico mediante la identificación de perfiles, no necesariamente guardan todas las características de la población de donde fueron obtenidas y es imposible calcular cuál es el grado de error que tienen. Este tipo de muestras se utiliza con fines exploratorios, cuando no se pretende inferir los resultados a todo el colectivo (López Romo 1998). Los datos recogidos de muestras no probabilísticas son analizados con las herramientas que ofrece la estadística descriptiva.

Entre las técnicas de muestreo no probabilístico, Cea D’Ancona (2004) menciona el muestreo estratégico o de juicio y el muestreo circunstancial. El muestreo estratégico (de juicio) "depende de la creencia de que [las unidades de población] pueden aportar información de interés o relevante para los objetivos del estudio" (Cea D'Ancona 2004: 171). En muestras muy pequeñas, por ejemplo inferiores a 30 casos, el muestreo estratégico puede favorecer la consecución de información más relevante que la obtenida de una muestra del mismo tamaño extraída al azar de la población de estudio. El muestreo circunstancial (en el estudio participan sólo los voluntarios que quieren participar) se guía por la facilidad de acceso a la unidad de observación. Este tipo de muestro incluye una variedad que es el muestreo de bola de nieve en la cual "las unidades de la muestra van escogiéndose, de forma sucesiva, a partir de las referencias dadas por los sujetos a los que ya se ha accedido" (Cea D'Ancona 2004: 172). Este tipo de muestreo resulta de gran utilidad en encuestas a población marginal. Cea D’Ancona apunta además que 
los muestreos no probabilísticos son muy comunes cuando se trata de realizar un pretest del cuestionario y en los estudios en los que la carencia de recursos (tesis doctorales e investigaciones académicas frente a investigaciones comerciales) y la ausencia de marcos muestrales fiables imposibilitan la práctica de alguno de los muestreos probabilísticos.

En cambio, para que una muestra sea estadísticamente representativa (probabilística), tiene que cumplir con dos principios fundamentales (López Romo 1998):

(1) Todos los elementos del colectivo deben tener probabilidad de ser incluidos en la muestra. En este caso, el investigador debe asegurarse de que todos los elementos que constituyen la población tengan posibilidad de ser elegidos. Si, por la forma de seleccionar la muestra, se elimina la posibilidad de que alguno de los elementos que compone el universo sea elegido, entonces se anula, al mismo tiempo, la posibilidad de representar las características de ese elemento (las características del elemento eliminado no están representadas).

(2) La probabilidad de cada elemento de ser incluido en la muestra debe ser conocida. Dicha probabilidad se define como las oportunidades que tiene un elemento de ser elegido. En cambio, el valor opuesto de la probabilidad se define como el factor de representatividad de cada elemento; es decir, el número de casos que representa. Por lo tanto, este requisito de una muestra representativa se cumple, si se conoce el factor de representatividad.

Como vemos, existe un prerrequisito para el segundo principio arriba mencionado que se traduce en la necesidad de conocer, al menos de una manera orientativa, el tamaño de la población para poder definir el factor de representatividad de los elementos de la muestra. Este requisito, en muchas ocasiones, no está explicitado en el caso de universos hipotéticos (distintos de poblaciones) que carecen de definición numérica de las características de la población estudiada (no se conoce su tamaño ni siquiera de manera orientativa).

En cuanto al tipo de selección de muestras probabilísticas, las técnicas más usadas son la técnica aleatoria simple, aleatoria PPT (proporcional al tamaño) y selección por cuotas (muestras proporcionales) (López Romo 1998: 58).

\section{Estudios con encuesta de tipo social en Traductología}

En este apartado presentamos varios estudios realizados en Traductología que usan la encuesta como método de investigación social. Nuestra recopilación 
no pretende ser exhaustiva, tan sólo queremos dar algunos ejemplos, tal vez los más ilustrativos, de este nuevo género metodológico en la disciplina. Nuestra revisión abarca estudios publicados entre 1992 y 2005.

Hemos agrupado estos estudios en dos grandes bloques temáticos: encuestas orientadas a mejorar la adecuación entre la formación y el mercado laboral (Golden et al. 1992, Mackenzie 2000, Li 2000, Calvo Encinas 2004, Lim 2005) y encuestas cuyo objetivo es recoger la percepción de los colectivos en torno a la calidad de la interpretación y subtitulación (Chiaro y Nocella 2004, Widler 2004).

Para la selección de los estudios, hemos seguido los siguientes criterios:

(1) Variedad tipológica, con el fin de proporcionar ejemplos para las diferentes formas de encuesta, recogidas por López Romo (ver Tabla 1); así obtenemos un mapa muy variado en cuanto a los principales elementos de diseño de las encuestas: objeto de estudio, objetivos, hipótesis, población, muestreo, área geográfica estudiada, etc.

(2) Patrocinio. Todas las encuestas aquí analizadas se realizaron en el ámbito académico y no mercantil. Las encuestas patrocinadas por alguna entidad mercantil y de acceso restringido no suelen tener un marco teórico elaborado, ya que se considera que el patrocinador sustituye el marco teórico con sus propios objetivos e intereses; el marco teórico está, pues, implícito en el encargo de la encuesta. Como ejemplo de una encuesta reciente encargada fuera del ámbito académico nos puede servir la encuesta de la Agrupación de Centros especializados en Traducción (ACT 2005).

(3) Centralidad y autosuficiencia del método de la encuesta. Todos los estudios seleccionados se sustentan en este método. De esta manera quedan descartados los estudios que utilizan la recogida de datos mediante cuestionario, por ejemplo en la fase preparatoria, para seleccionar la muestra del estudio y asegurarse de que todos los sujetos cumplan los mismos criterios (p.e. PACTE 2005 y 2008). Tampoco comentaremos estudios basados en entrevistas estructuradas (p.e. Hermans y Lambert 1998).

En las publicaciones que citamos, los estudios realizados no aparecen descritos de manera uniforme dado que a veces faltan datos metodológicos o los que hay resultan difíciles de interpretar unívocamente. La amplitud y la profundidad de análisis de cada estudio son igualmente muy dispares. Por lo tanto, y a pesar del afán de unificar nuestra presentación, no siempre ha sido posible estructurarla del mismo modo. 


\subsection{Encuestas para adecuar la formación a la realidad laboral}

2.1.1. Golden, Hurtado Albir y Piqué (1992)

A partir de las fuentes bibliográficas consultadas, la primera encuesta en el ámbito de Traductología en España fue iniciada en el año 1988 por la Escola Universitària de Traductors i d'Intèrprets (EUTI) de la Universitat Autònoma de Barcelona (Golden et al. 1992).

El estudio formaba parte de un proyecto más amplio cuyo objetivo era describir la situación de la traducción e interpretación en Cataluña para conocer mejor este mercado y adaptar los nuevos planes universitarios que entraban en vigor en el curso académico 1992-1993 (transformación de "diplomaturas" en licenciaturas de $1^{\circ}$ y $2^{\circ}$ ciclo). Sus objetivos eran descriptivos (conocer la situación del mercado de la traducción e interpretación en función de la oferta y la demanda existentes y estudiar las características específicas de la traducción e interpretación en un medio bilingüe), pedagógicos (dar pautas para la elaboración de nuevos planes de estudios de la licenciatura de TI) y pragmáticos (elaborar una base de datos de traductores e intérpretes de Cataluña).

El estudio constaba de dos encuestas dirigidas a dos colectivos y realizadas en dos años distintos:

(1) Primer colectivo: empleadores de traductores, usuarios de servicios de traducción e interpretación, representantes de la demanda laboral del sector público y privado (ayuntamientos, administración pública, autonómica, estatal, extranjera, empresas públicas y privadas, asociaciones profesionales y editoriales).

(2) Segundo colectivo: traductores e intérpretes de Cataluña, como representantes de la oferta laboral.

Los investigadores decidieron realizar la segunda encuesta (entre los traductores) debido a una tasa muy baja de respuesta obtenida en el primer estudio. La tasa de respuesta del primer colectivo (empleadores de traductores) fue de un 3,96\%; y la tasa de respuesta del segundo colectivo (traductores) de un $23,53 \%$.

La encuesta consistía en ambos casos en el envío por correo tradicional de un cuestionario con preguntas de elección múltiple y preguntas abiertas, acompañado de otros documentos. Creemos que se debe resaltar el interés por crear una base de datos de traductores e intérpretes de Cataluña. Fue también una de las primeras señales en la disciplina de la necesidad de crear bases de datos actualizadas. 
Para seleccionar la muestra no se aplicó ninguna técnica de muestreo probabilístico. Se recopilaron previamente los datos de las entidades de interés en una base de datos (programa electrónico dBASE IV). La encuesta no era anónima y recogía los datos de la entidad encuestada pero no los datos del informante y de su función en esta entidad.

Se estudió el perfil del traductor e intérprete desde el punto de vista de los usuarios de la traducción en relación con los siguientes temas: frecuencia de la necesidad de la traducción e interpretación; tipos de textos más traducidos; situaciones y áreas de conocimiento donde más se necesita la interpretación; frecuencia de recurrir a los profesionales de traducción e interpretación frente a los no expertos; y situación del traductor e intérprete en las empresas privadas y organismos públicos.

En cuanto a la descripción de la oferta se abordaron los siguientes temas: perfil de los traductores e intérpretes (formación y especialización); grado de dedicación profesional; situación laboral; tipo de textos más traducidos; situaciones y temas que más requieren de la interpretación; combinaciones lingüísticas más frecuentes; distribución de lenguas maternas (castellano versus catalán).

Los resultados se analizaron en términos de porcentajes para los dos colectivos. Entre otras conclusiones se formularon las siguientes afirmaciones descriptivas:

(1) Predominancia de la traducción escrita frente a la interpretación (el 96\%).

(2) Falta de profesionales dedicados sólo a la interpretación (mayoritariamente, se combina con la modalidad escrita).

(3) Alto volumen de textos literarios y ensayos traducidos ante todo al catalán frente a textos de perfil no literario (traducciones técnicas, científicas, administrativas, audiovisual, etc.).

(4) Predominancia de temas relacionados con la medicina, la política y la economía en las interpretaciones.

(5) Gran demanda de traducciones entre el castellano y el catalán (combinación de lenguas no reconocida para fines laborales).

(6) Precariedad de la profesión del traductor y del intérprete tanto por falta del debido reconocimiento de esta profesión por las entidades iniciadoras de traducciones como por el bajo índice de adhesión a asociaciones profesionales de traductores e intérpretes 


\subsubsection{Mackenzie (2000)}

La encuesta realizada por Mackenzie en la Universidad de Turku se enmarca en un proyecto más amplio: Practical Orientation of Studies in Translation and Interpreting (POSI) (Mackenzie 2000), que surgió de la necesidad de mejorar la preparación profesional de los graduados en traducción e interpretación en Alemania. Luego el proyecto se extendió a varios países europeos. Por parte de Finlandia, el grupo investigador POSI estuvo formado por cinco representantes de universidades finlandesas que ofrecen la titulación en traducción e interpretación, tres representantes de la Finnish Associations of Translators and Interpreters, Translation Companies and Professional Translators y un representante de los usuarios de servicios de traducción, Nokia Communications.

La técnica de recogida de datos fue una encuesta con cuestionario. Como en los estudios anteriores de este tipo (Golden et al. 1992; Hermans y Lambert 1998), se investigaron dos colectivos distintos:

(1) Primer colectivo: usuarios de servicios de traducción representados por las 48 compañías más grandes de Finlandia (elegidas de una lista de 500), 53 PYMEs exportadoras e importadoras de la región de Turku, 11 ministerios, una oficina de información del Consejo del Estado y las oficinas de administración de 10 ayuntamientos de las ciudades más grandes de Finlandia.

(2) Segundo colectivo: proveedores de servicios de traducción, representados por 22 empresas de traducción (20 de ellas miembros de Finnish Association of Translation Companies), 212 traductores e intérpretes (miembros de Finnish Association of Translators and Interpreters; entre ellos 110 residentes de Finlandia y 102 residentes de otros países europeos; algunos de ellos colaboradores de la Unión Europea). Además, se enviaron tres cuestionarios a los supervisores de servicios de traducción e interpretación de la Unión Europea para que distribuyesen el cuestionario entre los traductores implicados.

Tal como se desprende de la publicación, no se realizó ninguna técnica de muestreo probabilístico. Se efectuó una prueba piloto del cuestionario entre los estudiantes de traducción.

El objetivo de la encuesta era recoger opiniones de las dos poblaciones mencionadas en cuanto a:

(1) Qué competencias se consideran más importantes actualmente en el mercado desde el punto de vista de los usuarios y de los proveedores.

(2) Qué competencias, en opinión de los usuarios, faltan en la preparación profesional actual de los traductores recién contratados. 
(3) Si los traductores están satisfechos de la formación que han recibido y qué les ha hecho falta en la formación recibida.

(4) Cómo se podría mejorar la formación de los traductores, según los usuarios de los servicios de traducción.

(5) Qué tipo de formación continuada se podría proponer a los traductores.

Las preguntas incluidas en los dos cuestionarios se referían directamente a los conocimientos y competencias relacionadas con las tareas traductoras sin contextualizar, operativizar o ejemplificar estos conceptos. Además, siendo una investigación orientada a fines didácticos, se centraba en traductores formados en centros especializados, dejando de lado los traductores autodidactas o procedentes de otra formación.

De este estudio queremos resaltar dos puntos, a nuestro parecer, muy interesantes:

(1) Conciencia del doble estatus de las empresas de traducción en el mercado que, siendo intermediarios entre los traductores y el receptor final, son al mismo tiempo proveedores de servicios de traducción (frente al usuario final) y usuarios de los servicios de traducción (frente a los centros de formación de los traductores).

(2) Conciencia de la función del informante dentro de la organización, ya que los autores del estudio tuvieron en cuenta la jerarquía laboral existente dentro de las empresas e instituciones.

\subsubsection{Li (2000)}

En octubre del año 1998, Li (2000), investigador de la Chinese University of Hong Kong, realizó un estudio entre los traductores profesionales para indagar sus necesidades de trabajo, conocer su opinión en cuanto a la formación en traducción recibida y recabar ideas en torno a los perfiles de formación continuada en traducción que más se necesitan. Estos tres objetivos específicos se englobaban en un objetivo principal que consistía en conocer las necesidades sociales, presentes en el mercado laboral, con vistas a mejorar la formación en traducción.

El estudio constaba de dos partes:

(1) Una encuesta dirigida a 42 traductores profesionales de Hong Kong: 24 de ellos eran traductores oficiales del gobierno chino, 16 traductores de empresas privadas (dedicadas a la traducción o no) y 2 traductores de policía.

(2) Entrevistas semiestructuradas con 12 traductores procedentes de la misma muestra de la encuesta. 
Para la selección de la muestra de la encuesta no se siguió ninguna técnica probabilística de muestreo. Para las entrevistas semiestructuradas se eligieron 12 sujetos que se diferenciaban en cuanto a la edad, experiencia, formación, y por lo tanto presentaban todos los perfiles socioprofesionales incluidos en la muestra.

La encuesta proporcionaba datos cuantitativos y las entrevistas semiestructuradas en profundidad, datos cualitativos. Todos estos datos fueron analizados conjuntamente siguiendo un principio de inducción analítica, o sea sin plantear hipótesis de trabajo previas al estudio sino analizando los datos $a$ posteriori y sacando conclusiones globales descriptivas, que fueron presentadas en forma de rangos y porcentajes.

El cuestionario contenía 44 preguntas reagrupadas en tres partes: información personal sobre el informante; experiencia en traducción e interpretación; y percepción y evaluación de la formación en traducción e interpretación. Las preguntas eran cerradas (de elección múltiple) y abiertas.

De 65 cuestionarios enviados se recibieron 42 cuestionarios (tasa de respuesta $64,6 \%$, una tasa muy alta). Las entrevistas semiestructuradas (con preguntas estandarizadas, abiertas y libres) fueron grabadas y transcritas.

Se estudió las necesidades sociales de los traductores en torno a las siguientes cuestiones:

(1) ¿Cuál ha sido el curso más útil durante la formación universitaria en traducción e interpretación? Respuesta predominante: lengua y literatura inglesa.

(2) ¿En qué área se sentía mejor preparado al empezar la profesión de traductor? Respuesta predominante: competencia en lengua inglesa y china.

(3) ¿Cuál es el mayor reto en el trabajo de traductor? Respuesta predominante: encontrar el estilo correcto para cada género textual en inglés y la interpretación.

(4) ¿Qué cambios nota en el mercado de traducción? Respuestas predominantes: más traducciones de chino a inglés; más traducciones sintéticas; y más traducciones relacionadas con China continental.

(5) ¿Qué es lo que más le gustaría aprender si tuviese la oportunidad de participar en una formación in-service? Respuesta predominante: literatura y lengua inglesa.

La discusión en torno a los resultados obtenidos se centra sobre todo en la última pregunta y traza posibles vías de desarrollo para una formación orientada a la ejecución de encargos de traducción reales. 


\subsubsection{Calvo Encinas (2004)}

En el año 2000 Calvo Encinas (2004), en el marco de estudios de doctorado en la Universidad de Granada, realizó una encuesta en torno a la interpretación social.

La encuesta fue de tipo exploratorio y el enfoque que se le dió era cualitativo. El terrirorio de estudio fue la provincia de Toledo (España). La población estudiada, los funcionarios de Administración Pública.

Su objetivo general era estudiar las necesidades y percepciones de la propia Administración respecto a la interpretación social. Entre los objetivos específicos del estudio se cita:

- Analizar si los encuestados reconocen la necesidad de contar con el apoyo permanente y/o institucionalizado de intérpretes en el ámbito provincial, que pudiera garantizar un mejor servicio al actual.

- Recoger las impresiones de los funcionarios de la Administración del Estado en la provincia de muestra [Toledo] con respecto a las dificultades que ellos mismos experimentan a la hora de atender a los individuos de habla no hispana.

- Analizar el conocimiento que existe actualmente en torno al concepto de interpretación social.

- Definir el perfil del intérprete social o profesional lingüístico ideal en cada caso. (Calvo Encinas 2004: 8)

Se siguieron dos criterios para elegir la provincia de Toledo como representativa de España: ser una de las 10 provincias españolas con mayor índice de inmigración y una de las provincias de interior con mayor afluencia turística.

La muestra fue de 60 informantes funcionarios procedentes de distintos ámbitos de la Administración Pública (Jefatura Superior de Policía, Justicia, Delegación de Educación, Bienestar Social, INEM, Centros Hospitalarios, Agencia Tributaria, Ayuntamiento de Toledo, Sindicatos, varias ONGs). No se aplicó ninguna técnica de muestreo probabilístico. Para la elección de los informantes se siguieron dos criterios: que los funcionarios mantuvieran un trato real y directo con el público y que tuvieran un conocimiento exhaustivo de la situación, sin atender especialmente a su puesto dentro de la jerarquía administrativa.

La técnica de recogida de datos fue un cuestionario que el encuestador rellenaba en presencia del encuestado durante su trabajo de campo. Se realizaron varios diseños piloto para conseguir un cuestionario válido. El cuestionario definitivo constaba de tres bloques de preguntas: el primero describe el perfil del entrevistado; el segundo pretende analizar el estado de la cuestión 
según el encuestado; y el tercero pretende concretar las respuestas en ámbitos laborales más específicos (Fuerzas de Seguridad del Estado y Centros de Salud).

De este estudio cabe resaltar una importante labor previa de documentación sobre las entidades encuestadas, así como el interés por el puesto de trabajo del informante. Una de las preguntas del primer bloque recoge información sobre el puesto del funcionario, si bien parece que, para el análisis de los datos, las categorías de estos puestos no son consideradas relevantes.

Entre otras cuestiones, los resultados ponen de manifiesto el desconocimiento por parte del $100 \%$ de la muestra del concepto de "interpretación social", "interpretación de enlace" e "interpretación comunitaria".

\subsubsection{Lim (2005)}

En Seúl (Corea del Sur) Lim realizó una encuesta, a partir de marzo del 2004, entre los estudiantes de primer y segundo curso de interpretación coreano - inglés de la Graduate School of Interpretation and Translation de Hankuk University of Foreign Studies (Lim 2005). Lim se basó en un estudio anterior de Li (2002, cit. en Lim 2005).²

El estudio tenía un enfoque exploratorio, sin ninguna técnica de muestreo probabilístico, con una alta tasa de respuesta, al tratarse de una población de estudiantes.

La encuesta se realizó entre estudiantes de primero y segundo curso para investigar sus expectativas, opiniones y necesidades relacionadas con los estudios de interpretación y, de esta manera, contribuir a mejorar los diseños curriculares de la escuela de traducción.

Del primer curso se obtuvieron 44 cuestionarios rellenados de 56 distribuidos (tasa de respuesta 79\%); del segundo curso se obtuvieron 44 respuestas de 52 cuestionarios repartidos (tasa igual a 85\%).

El cuestionario, además de contener preguntas relacionadas con las percepciones y opiniones personales de los sujetos, introducía preguntas relativas a sus familias. Uno de los resultados más evidentes de este estudio fue la confirmación de que hay muchas más mujeres que hombres estudiando interpretación. La profesión de intérprete representa para las mujeres de Corea un

2. En el presente artículo presentamos un estudio con encuesta (cf 2.1.3.), combinada con entrevistas semiestruturadas, realizada por Li en el año 1998 ( Li 2000) en la cual la población estudiada fueron traductores. Lim (2005: 175) menciona otro estudio de Li (2002) en el cual participaron alumnos de traducción y que fue realizado usando grupo de discusión, encuesta y entrevistas. 
buen medio de ascenso social. En cambio, los hombres no están interesados por esta profesión porque actualmente no posee el prestigio social que les pueda motivar suficientemente.

\subsection{Encuestas para recoger la percepción de la calidad}

\subsubsection{Chiaro y Nocella (2004)}

En octubre del mismo año 2000, Chiaro y Nocella (2004) de la Universidad de Bolonia (Italia) lanzaron una encuesta global a través de Internet entre intérpretes profesionales para investigar su percepción de los criterios de calidad de la interpretación. Cabe señalar que los autores realizan una elaboración previa del marco teórico muy buena.

El estudio parte de una revisión de tres tipos de investigaciones realizadas anteriormente por otros autores y dedicadas a la percepción de la calidad en la interpretación: análisis de textos de interpretaciones transcritas; estudios de campo con usuarios finales (asistentes y ponentes de conferencias); investigaciones empíricas con intérpretes y estudiantes de interpretación. Del último grupo de trabajos realizados, Chiaro y Nocella seleccionaron el de Bühler (1986, cit. en Chiaro y Nocella 2004: 282), que pretendía recoger la opinión entre los intérpretes sobre un conjunto de diecisiete criterios lingüísticos y extralingüísticos de calidad.

La muestra fue de 286 intérpretes, cuyas direcciones de correo electrónico se encontraron en páginas web. No se aplicó ninguna técnica de muestreo probabilístico. El 44\% de informantes provenían de Europa Occidental, 27\% de América Central y del Sur, 19\% de América del Norte, 5\% de Europa del Este y 5\% del resto de países.

El instrumento de recogida de datos fue un breve cuestionario en formato electrónico con dos bloques de preguntas:

(1) El primer bloque reunía las preguntas en torno a la edad de los encuestados, lugar de nacimiento, formación y experiencia laboral.

(2) El segundo bloque incluía preguntas basadas en los criterios de calidad de interpretación de Bühler así como en resultados de varias consultas y entrevistas con intérpretes profesionales. Los encuestados tenían que dar su opinión en cuanto al rango que ocupa, según ellos, cada criterio (desde el menos importante hasta el más importante). Los criterios de calidad estaban agrupados en nueve criterios lingüísticos y ocho extralingüísticos.

Los resultados fueron analizados en términos de porcentajes pero también se aplicó un análisis de escala multidimensional (multidimensional scaling 
MDS) usando el software STATISTICA para Windows, que permite crear un tipo de mapa evaluador y perceptivo bidimensional, al estilo de una configuración geométrica. El mapa generado representa el resumen de cómo los informantes perciben los criterios lingüísticos de calidad en interpretación, distribuidos según dos escalas: el eje horizontal de la dimensión de calidad; y el eje vertical de la dimensión estructural (Chiaro y Nocella 2004: 290).

El estudio permitió seleccionar tres criterios lingüísticos de calidad de interpretaciones, indicados con mayor frecuencia por los intérpretes: la adecuación al original; la integridad de la información y su cohesión lógica, que fueron clasificados a posteriori como criterios cruciales de calidad. Los criterios menos mencionados fueron nombrados criterios de "adorno" (timbre de la voz, acento, estilo).

En este estudio, la recogida de datos con un cuestionario fue completamente automatizada. Podemos decir que los autores validaron a gran escala el uso del ordenador y de Internet para las encuestas en nuestra disciplina. Gracias a la aplicación de las nuevas tecnologías, los autores pudieron doblar la tasa de respuesta entre los encuestados (la tasa de una encuesta tradicional por correo es de unos 10-15\%), economizar gastos y tiempo, mejorar la calidad de cuestionarios recibidos (el formato electrónico no permitía reenviar un cuestionario mal rellenado), y recopilar datos de los cinco continentes. Para ello, los autores tuvieron que apoyarse en especialistas en informática y en bases de datos para realizar su tarea investigadora. Chiaro y Nocella reconocen que su investigación fue multidisciplinaria porque tuvieron que cooperar con especialistas de otras disciplinas para llevar a cabo la encuesta.

\subsubsection{Widler (2004)}

Widler (2004) realizó una encuesta de tipo exploratorio entre agosto y septiembre del año 2002 en Viena (Austria). Su estudio formó parte de un proyecto de investigación más amplio, "Literary translation as multimedial communication", organizado por la Facultad de Traducción de la Universidad de Viena en colaboración con la Fundación Austriaca de Ciencia (FWF). Aparte de su situación en un marco de estudio más grande, Widler reconoce que colaboraron también especialistas en análisis estadísticos y otros compañeros que le ayudaron a realizar las entrevistas.

Se estudiaron 100 espectadores de 19 películas en 9 lenguas originales (todas subtituladas en alemán) en 7 cines de Viena y 2 festivales de cine de verano. Los datos de las películas, cines y festivales provenían de Der Standard (un diario austriaco) y de Falter (una guía semanal de eventos culturales de Viena). No se aplicó ninguna técnica de muestreo probabilístico. 
El estudio se proponía explorar y describir el universo de los espectadores de películas subtituladas en Viena: ¿quiénes son?, ¿qué edad tienen?, ¿cuál es su formación y profesión?, ¿con qué frecuencia ven películas subtituladas?, ¿están contentos con las subtitulaciones?

La técnica de recogida de datos fue un cuestionario administrado personalmente por el entrevistador. Los encuestadores se acercaban a las personas que acababan de comprar entradas a las películas subtituladas y en el tiempo entre la compra y la película rellenaban el cuestionario con las respuestas que proporcionaba el entrevistado.

El valor de este estudio radica en realizar uno de los primeros estudios de audiencia en nuestra disciplina y en plantear claramente hipótesis de trabajo. Cuatro de ellas fueron confirmadas: formación universitaria de los espectadores (51\%); frecuencia en ir al cine (65\%; mínimo varias veces al mes); satisfacción de la calidad de la subtitulación (51\%) y expectativa de que se proyecten en los cines más películas subtituladas (61\%). Una de las hipótesis refutadas concernía la edad de los espectadores, ya que sólo un 48\% tenía de 31 a 50 años. La segunda hipótesis que resultó falsa se refería a la motivación de los espectadores para ver películas subtituladas en los cines; muchos admitieron que les motivaba la necesidad de mejorar el nivel de lengua extranjera. No se esperaba recibir puntuación tan alta para esta hipótesis.

\section{Características metodológicas de los estudios realizados}

En este apartado concluimos con las principales características metodológicas de las encuestas que acabamos de presentar. Hemos agrupado dichas características en los siguientes apartados: diseño general del estudio, población y muestra e instrumento de recogida de datos.

\subsection{Aspectos relacionados con el diseño general del estudio}

La Traductología constituye, hoy en día, un nuevo campo de uso de la encuesta y una nueva área de aplicación de sus resultados, que se suma a los diferentes campos de aplicación existentes hasta ahora (mercadotecnia, medios de comunicación y publicidad, opinión pública, salud, demografía, etc.).

\section{Temas abordados}

Los estudios realizados abarcan tanto la traducción escrita (Golden et al. 1998, Mackenzie 2000, Li 2000) como la interpretación (Calvo Encinas 2004, Chiaro y Nocella 2004, Lim 2005) y la traducción audiovisual (Widler 2004). 
Los temas que se han investigado son temas de mucha actualidad y propios del desempeño laboral de la profesión de traductor e intérprete. Van desde las necesidades del mercado para adecuar los contenidos pedagógicos, la preparación profesional de los graduados y su inserción laboral, pasando por la situación de servicios de traducción y valor de ventas, hasta el concepto de calidad de distintas modalidades de la traducción y de su prestación mercantil en forma de servicio.

\section{Objetivos perseguidos}

Los objetivos de todas las encuestas presentadas son de interés académico y descriptivo pero, además, algunos estudios persiguen una aplicación pedagógica (Golden et al 1998, Mackenzie 2000, Li 2000, Lim 2005). Las encuestas de Calvo Encinas (2004), Widler (2004) y Lim (2005) tienen un enfoque descriptivo y claramente exploratorio. Por otro lado, dos encuestas se proponen cumplir con objetivos más específicos: un objetivo pragmático de creación de un directorio de traductores e intérpretes de Cataluña (Golden et al 1998) y un objetivo metodológico, la validación del ordenador como instrumento de recogida de datos (Chiaro y Nocella 2004). Es muy común que se combinen en el mismo proyecto varias finalidades y tipos de objetivos, ya que en sí las encuestas suelen ser largas y costosas.

\section{Enfoque metodológico y marco teórico}

Las encuestas de Calvo Encinas (2004) y Lim (2005) reconocen su carácter exploratorio y por lo tanto no se sustentan en un marco teórico elaborado. Muy diferente es el trabajo de Chiaro y Nocella (2004), que parte de una elaboración teórica bien fundamentada. Widler (2004) no presenta ningún marco teórico pero formula sus hipótesis de trabajo, que se ven contrastadas a lo largo del estudio. Li (2000) reconoce que aplica un método inductivo y que, por lo tanto, su marco teórico se va construyendo a lo largo del proceso investigador.

En cuanto al grado de elaboración del marco teórico, el estudio de Golden, Hurtado Albir y Piqué (1992) y el de Mackenzie (2000) tienen un marco teórico implícito, como sucede en las encuestas de tipo mercantil. Supuestamente, el marco teórico del que parten es el relativo al mercado laboral y a la economía del sector de servicios pero no se elaboran teóricamente dichos conceptos, como tampoco se profundiza en ninguna corriente económica y laboral concreta. 
Tipo de triangulación

En las encuestas de Golden, Hurtado Albir y Piqué (1992) y en la de Mackenzie (2000) observamos que los dos colectivos estudiados (representantes de la demanda y de la oferta laboral) se reflejan mutuamente y dan su opinión acerca del contexto de actuación que comparten, o sea, del mercado laboral y del mercado de los servicios de traducción e interpretación. A este efecto de contrastación de opiniones lo denominamos efecto "espejo". Según la tipología de triangulación propuesta por Denzin (1970), éste sería el caso de una triangulación teórica. Cabe señalar que la baja tasa de respuesta de uno de los colectivos estudiados se compensa gracias a la combinación de dos tipos de colectivos (Golden et al. 1998). Generalmente, la tasa de respuesta más baja proviene de los empleadores de los traductores, representantes de la demanda laboral. Para paliar las deficiencias en las respuestas recibidas de este colectivo, se recoge la información de los traductores (representantes de la oferta laboral).

La encuesta de Li (2000) es el único estudio en que se realiza una triangulación de tipo metodológico (Denzin 1970, Flick 2004), combinando la estrategia cuantitativa (encuesta) con la cualitativa (entrevistas semiestructuradas).

En el estudio de Calvo Encinas (2004), los datos principales obtenidos de la encuesta se completan con datos adicionales obtenidos de la contextualización previa (análisis de fuentes documentales secundarias), sin llegar a obtener una triangulación teórica o metodológica.

Chiaro y Nocella (2004) tampoco aplican triangulación; en cambio, realizan un tratamiento estadístico de datos más avanzado (análisis multivariante).

\section{Alcance geográfico}

Las encuestas presentadas tienen un diverso alcance: local, regional y mundial. Se realizan en muchas zonas, países y continentes: España (Golden et al. 1992, Calvo Encinas 2004), Finlandia (Mackenzie 2000), Austria (Widler 2004), Hong-Kong (Li 2000), Corea del Sur (Lim 2005) o Italia con una prospección mundial (Chiaro y Nocella 2004).

Ahora bien, en estos estudios no se comparan resultados de diferentes áreas geográficas. En este sentido, cabe considerar que, para el caso de diseños de encuestas muy amplias, entre varios países o continentes, se tiene que tener siempre en cuenta la correcta adaptación del estudio y de su instrumento (cuestionario) a las diversas sociedades en que se realiza el trabajo de campo (Behling y Slaw 2000). 


\section{Carácter interdisciplinario}

Debido a los costes y al número de personal involucrado en estudios por encuesta, varias de las encuestas aquí comentadas fueron realizadas en el marco de proyectos más amplios, universitarios, regionales o europeos subvencionados (Golden et al. 1992, Mackenzie 2000, Widler 2004). No es raro que participe en la organización y ejecución de una encuesta más de un centro universitario o más de un país.

Siendo caro, lento y laborioso, requiere un trabajo de equipo (Chiaro y Nocella 2004), que en muchas ocasiones reúne a expertos de diferentes disciplinas: profesionales de estadística, psicólogos, entrevistadores, redactores, documentalistas, analistas de contenidos y diseñadores gráficos.

\subsection{Aspectos relacionados con la población y la muestra}

\section{Población}

Se han podido identificar cuatro grupos de poblaciones: estudiantes de traducción e interpretación (Lim 2005); traductores e intérpretes (Golden et al. 1992, Mackenzie 2000, Li 2000, Chiaro y Nocella 2004); usuarios finales de la interpretación (Calvo Encinas 2004, Widler 2004); empleadores de traductores e intérpretes (Golden et al. 1992, Mackenzie 2000), teniendo en cuenta el doble papel de proveedores y usuarios de las empresas de traducción. Como hemos mencionado en el apartado anterior, el punto de vista de la población de los traductores y de sus empleadores fue objeto de triangulación en dos encuestas (Golden et al. 1992, Mackenzie 2000).

Según la terminología propuesta por López Romo (1998; Tabla 1), las unidades estudiadas de estas encuestas se pueden desglosar en usuarios, audiencia, grupos laborales y profesionales. Se observa que el uso de la encuesta en el análisis de las organizaciones propias del ámbito de la traducción (empresas de traducción, otras empresas mercantiles, instituciones públicas, etc.) no es muy común (Hermans y Lambert 1998).

La definición y delimitación de las poblaciones estudiadas en las encuestas refleja la concepción que tienen sus autores en cuanto a la estructuración del mercado laboral y de los servicios de traducción (sector público y sector privado; empleadores, traductores y usuarios; la doble función de las empresas de traducción).

Dichas poblaciones están definidas de manera muy abstracta y, en este sentido, se asemejan a los universos hipotéticos mencionados por AnderEgg (1990: 179). Su carácter abstracto emana principalmente del hecho de que son poblaciones numérica y socialmente ilimitadas: en ningún sitio se 
menciona, aunque sea aproximadamente, el tamaño numérico de éstas y tampoco el tamaño de los perfiles teóricos que las constituyen. Por lo tanto, no se conoce ni la probabilidad de cada elemento de ser incluido en la muestra, ni su factor de representatividad (López Romo 1998).

La dificultad que ocasiona la definición de las poblaciones en nuestra disciplina ha sido mencionada por Neunzig (2002) en relación con los diseños experimentales:

Uno de los principales problemas aquí radica en determinar lo que uno entiende, p.e. bajo 'traductores experimentados', es decir, el universo que se quiere examinar, ya que no existe un criterio externo y aceptado comúnmente que lo defina. La definición del universo (y con ello la elaboración de la muestra) es determinante en el momento de la interpretación (siempre subjetiva) y de la extrapolación (sólo válida para el universo definido) de los datos. (Neunzig 2002: 83)

\section{Muestreo}

El muestreo aplicado ha sido de tipo teórico, exploratorio y no probabilístico. En el muestreo se han seguido siempre unos criterios de selección, pero no se ha procedido a ninguna técnica de muestro probabilístico y por lo tanto, desde el punto de vista estadístico, estas muestras no son representativas (López Romo 1998, Tabla 1). En consecuencia, no se ha podido aplicar ninguna operación de estadística inferencial para extrapolar los resultados a toda la población. ${ }^{3}$

Por consiguiente, las muestras obtenidas en las encuestas presentadas pueden llamarse de criterio y no probabilísticas. Aplicando la terminología de Cea D'Ancona (2004) podríamos decir que son muestras circunstanciales porque participan en la encuesta sólo voluntarios (Chiaro y Nocella 2004: 285). La falta de respuesta de representantes de algún perfil teórico no se complementa posteriormente con otros representantes del mismo perfil.

Las limitaciones para obtener una muestra estadísticamente representativa pueden tener sus orígenes en la carencia de marcos muestrales fiables y sólidos, previos al estudio (Golden et al. 1992). Como hemos podido comprobar en las encuestas presentadas, cada estudio de este tipo tiene la necesidad de construir su propio marco muestral ad hoc (base de datos previa de todas las unidades de la población), lo que contribuye a alargar el proceso de

3. Orozco (2002: 73-77) señala en este sentido que incluso en el campo de la investigación experimental en traducción escrita se dan casos de generalizaciones metodológicamente no fundamentadas porque están basadas en muestras no representativas. 
investigación y a elevar sus costes. Parecen muy escasas las investigaciones en nuestro campo que exploten bases de datos ya existentes, como sería por ejemplo la Muestra Continua de Vidas Laborales facilitada a los investigadores por la Diputació de Barcelona (Valero et al. 2008).

En este sentido, Vande Walle, desde una perspectiva económica, apunta que la falta de un organismo oficial encargado de recoger datos estadísticos y la falta de rigor metodológico en los estudios empíricos cuantitativos en nuestra disciplina imposibilitan la elaboración de una visión clara del mercado de traducción, su evolución y sus tendencias; y, por consiguiente, dificultan cualquier toma de decisión al respecto:

Premier constat: il n'existe toujours pas d'organe officiel qui se charge d'établir des statistiques fiables pour notre profession, dans une perspective strictement économique, permettant d'établir des comparaisons, de dégager des tendances, voire de planifier des formations. [...] Bien sûr, il existe des études empiriques. Mais l'économie moderne ne peut se satisfaire d'approximations, d'hypothèses plus ou moins vérifiées sur un échantillon plus ou moins représentatif, de chiffres plus ou moins bien compilés pour les besoins de la cause. Il suffit de voir le nombre d'enquêtes qui paraissent régulièrement et où les chiffres ne servent jamais qu'à justifier la nouvelle version de la mémoire de traduction $X$ ou l'apparition du logiciel $Y$. Tous ces chiffres, toutes ces études [...] faites au cas par cas, n'ont aucun intérêt scientifique. (Vande Walle 2006: 81)

A diferencia de este autor pensamos que los estudios realizados en Traductología sí tienen un interés científico, aunque pueden carecer de interés económico, ya que no proporcionan suficiente fiabilidad estadística para poder generalizar los resultados a toda la población estudiada y asumir las decisiones económicas que se tomarían partiendo de ellos.

Una posible solución a este problema sería potenciar la recopilación y actualización de estas bases de datos de sujetos y entidades implicados en los estudios traductológicos. Uno de los grandes retos de la Traductología actual en el campo de la investigación empírica es impulsar investigaciones previas en torno al estudio y evaluación de marcos muestrales existentes. Otra solución sería cotejar más la definición de la población (y no trabajar con universos hipotéticos; Ander-Egg 1990) y así aumentar la posibilidad de obtener una muestra válida desde el punto de vista estadístico. Finalmente, sería conveniente elaborar perfiles teóricos adecuados a la población, también con una orientación aproximada de su representatividad numérica. 
3.3. Aspectos relacionados con el instrumento de recogida de datos

\section{Proceso de elaboración}

Todos los cuestionarios usados han sido objeto de un laborioso proceso de elaboración, pasando por varias etapas: fase exploratoria cualitativa, primera versión del instrumento, test, modificaciones y versión definitiva. Dicho proceso adquiere aún más relevancia si consideramos que todo cuestionario es una forma de comunicación específica, mediante diálogo (pregunta, respuesta), en el cual uno de los interlocutores no está presente (el que formula la pregunta); por lo tanto, no existe la posibilidad de asegurarnos de si el encuestado entiende todos los términos y cómo interpreta las preguntas que tiene que contestar (Low 1999).

\section{Estructura y formato de los cuestionarios}

Conviene señalar, de entrada, que no siempre se publican los instrumentos utilizados. En cuanto a los cuestionarios que se han podido consultar, contienen preguntas cerradas (de elección múltiple y con escala de Likert: "poco", "bastante", "mucho", etc.) pero también abiertas, que dificultan la estructuración posterior de datos. Se han aplicado instrumentos de tipo tradicional de papel y lápiz, y de tipo electrónico (Chiaro y Nocella 2004), autoadministrados o administrados por el encuestador (Calvo Encinas 2004, Widler 2004). Se han adaptado debidamente los cuestionarios usados en el mismo estudio a poblaciones diferentes, según las características específicas de cada colectivo. Algunos cuestionarios han sido acompañados de otras técnicas que proporcionaban datos de carácter cualitativo (entrevistas semiestructuradas, $\mathrm{Li}$ 2000; análisis de fuentes documentales, Calvo Encinas 2000). Parece bastante común que el primer bloque de preguntas se refiera a datos sociolaborales de los informantes. De ahí el gran interés de las encuestas como método de identificación y descripción de los colectivos.

Análisis de datos

Los datos recogidos con los cuestionarios han sido analizados con las herramientas matemáticas que ofrece la estadística descriptiva. Las medidas de resumen más usadas han sido las frecuencias expresadas en porcentajes. En este sentido, nos sumamos a la propuesta, expresada por Chiaro y Nocella (2004) de hacer mayor uso, en el ámbito de la Traductología, de medidas descriptivas como el rango, la moda y la mediana. 


\section{Conclusiones}

Como se ha podido observar con los siete estudios analizados, la Traductología ha incorporado en las últimas décadas los estudios por encuesta. A partir de los siete estudios analizados podemos deducir las siguientes conclusiones.

\section{Diseños investigadores sencillos}

Existe una gran variedad en cuanto a los aspectos relacionados con el diseño general del estudio; sin embargo, todos ellos comparten un diseño investigador sencillo, cuyas características metodológicas son:

- Un enfoque metodológico exploratorio y descriptivo (con escasez de enfoques explicativos y causales)

- Un carácter más bien cualitativo, sin llegar a aprovechar su potencial como método cuantitativo. Este fenómeno puede ser causado por las dificultades de obtener una muestra probabilística en nuestro campo.

- No suele ser muy frecuente la combinación (triangulación) de la encuesta con técnicas cualitativas (observación, entrevistas, grupo de discusión).

- $\quad$ Se realizan de manera unitaria en el tiempo (diseños transversales y no longitudinales).

\section{Estudio de colectivos socioprofesionales y muestras no probabilísticas}

Estos estudios recogen opiniones, valores, conocimientos, hábitos y necesidades de los diferentes grupos socioprofesionales implicados en el desempeño de la traducción y la interpretación. La contribución de la encuesta a la investigación traductológica de tipo social se ve reflejada también en que su uso plantea la necesidad de definir, delimitar y relacionar los diferentes colectivos implicados en el ejercicio de la traducción y la interpretación. La toma de conciencia de la estructuración social de dichos colectivos sirve, al mismo tiempo, para reforzar su consolidación.

Por otro lado, cabe resaltar que, dada la dificultad de obtener muestras estadísticamente representativas en el ámbito de la traducción e interpretación, los resultados numéricos no pueden ser inferidos al colectivo que la muestra representa; por consiguiente, resulta arriesgado partir de ellos para tomar decisiones. El propósito de los estudios con uso de encuesta en Traductología es principalmente estratégico (exploratorio), ya que se recogen informaciones de manera amplia y general, con el fin de diseñar y planificar actividades 
(p. e. la formación universitaria), pero no tienen un carácter concluyente para proyectos comerciales.

\section{Instrumentos de carácter cualitativo y bien elaborados}

El enfoque exploratorio de los estudios se refleja también en el carácter cualitativo de los instrumentos utilizados, ya que éstos contienen un número importante de preguntas abiertas. Por otro lado, cabe resaltar que han seguido un laborioso proceso de elaboración.

Como ya hemos señalado, algunos de estos instrumentos no se han publicado. Quisiéramos resaltar, en este sentido, la importancia de publicar los instrumentos de recogida de datos, y por consiguiente ponerlos al servicio de toda la comunidad científica, como hacen algunos estudios (Calvo Encinas 2004, Widler, 2004, Lim 2005, PACTE 2005, 2008). Desgraciadamente, estos casos no son muy numerosos.

Queremos finalizar resaltando que los estudios con encuesta en Traductología permiten establecer el contacto entre el mundo académico y profesional, ya que se interesan por temas actuales, relacionados con el ejercicio laboral y profesional de la traducción en sus más diversas manifestaciones. En este sentido, nuestra disciplina, además de incorporar un nuevo método de investigación, amplía su objeto de estudio para centrarse en los colectivos socioprofesionales implicados.

\section{Bibliografía}

ACT Agrupación de Centros especializados en Traducción. (2005) Estudio de situación del mercado español de servicios profesionales de traducción (2004).

ANDER-EGG, Ezequiel. (1990) Técnicas de investigación social. Buenos Aires: Humanitas.

ARnAU, Jaume. (1995) "Metodologies quantitatives en la investigació psicològica". En: Arnau, Jaume (ed.) 1995. Mètodes, dissenys i tècniques en investigació psicologica. ed. experimental. Barcelona: Universitat Oberta de Catalunya. pp. 4-64.

BEHLING, Orlando \& Kenneth S. Slaw. (2000) Translating questionnaires and other research instruments: problems and solutions. Thousand Oaks: Sage.

BERICAT, Eduardo. (1998) La integración de los métodos cuantitativo y cualitativo en la investigación social. Significado y medida. Barcelona: Ariel.

Cabo RAMÓn, Isabel de. (1998) "Métodes i tècniques". En: Cabo Ramón, Isabel de (coord.) \& Bernat Muniesa i Brito. 1998. Metodologia de les ciències socials i humanes. Barcelona: Universitat Oberta de Catalunya. pp. 115-146. 
Calvo EnCinAS, Elisa. (2004) "La Administración Pública ante la interpretación social: Toma de contacto en la provincia de Toledo", Puentes 4. pp. 7-16.

Cen D'Ancona, Ma Ángeles. (1996) Metodología cuantitativa. Estrategias y técnicas de investigación social. Madrid: Síntesis.

Cea D’Ancona, Ma Ángeles. (2004) Métodos de encuesta. Teoría y práctica, errores y mejora. Madrid: Síntesis.

CHIARO, Delia \& Guiseppe Nocella. (2004) “Interpreters' Perception of Linguistic and Non-Linguistic Factors Affecting Quality: A Survey through the World Wide Web". Meta 49:2. pp. 278-293.

CRESWELl, John W. (2003) Research design: Qualitative, quantitative, and mixed approaches. Thousand Oaks, CA: Sage.

DENZIN, Norman K. (1970) The Research Act. A Theoretical Introduction to Sociological Methods. Chicago: Aldine.

FLICK, Uwe. (2004) "Triangulation in Qualitative Research". En: Flick, Uwe; Ernst von Kardoff \& Inés Steinke (eds.) 2004. A Compagnion to qualitative research. Londres-Thousand Oaks-New Delhi: Sage Publications. pp. 178-183.

Golden, Sean; Amparo Hurtado Albir \& Ramon Piqué. (1992) "La traducció i la interpretació a Catalunya". Noves SL 17. pp. 14-17.

HERMANS, Johan \& José Lambert. (1998) "From Translation Markets to Language Management: The Implications of Translation Services". Target 10:1. pp. 113-132.

HurTado Albir, Amparo \& Fabio Alves. (2009) "Translation as a cognitive activity". En: Munday, Jeremy (ed.) 2009. The Routledge Companion to Translation Studies. Londres \& Nueva York: Routledge. pp. 54-73.

LEÓN, Orfelio G. \& Ignacio Montero. (1993) Diseño de investigaciones. Introducción a la lógica de la investigación en Psicología y Educación. Madrid: McGrawHill/ Interamericana de España.

LI, Defeng. (2000) "Tailoring translation programs to social needs: a survey of professional translators". Target 12:1. pp. 127-149.

LiM, Hyang-Ok. (2005) "Meeting Students' Expectations". Forum 1:3. pp. 175-204.

LÓPEZ RoMo, Heriberto. (1998) "La metodología de la encuesta". En: Galindo Cáceres, Luis Jesús (coord.) 1998. Técnicas de investigación en sociedad, cultura y comunicación. Méjico: Pearson Educación \& Addison Wesley Longman. pp. 33-73.

Low, Graham. (1999) "What Respondents Do with Questionnaires: Accounting for Incongruity and Fluidity". Applied Linguistics 20/4. pp. 503-533.

MACKENZIE, Rosemary. (2000) "POSI-tive Thinking About Quality in Translator Training in Finland". En: Beeby, Allison; Doris Ensinger \& Marisa Presas (eds.) 2000. Investigating Translation: Selected Papers from the $4^{\text {th }}$ International 
Congress on Translation, Barcelona, 1998. Amsterdam: John Benjamins. pp. 213-222.

Munday, Jeremy (ed.) (2009) The Routledge Companion to Translation Studies. Londres \& Nueva York: Routledge.

NeUnZiG, Wilhelm. (2002) "Estudios empíricos en traducción: apuntes metodológicos". Cadernos de Tradução X 2002:2, pp. 75-96.

OROZCO JUTORÁN, Mariana. (2002) "Revisión de investigaciones empíricas en traducción escrita”. TRANS 6. pp. 63-85.

PACTE. (2005) "Investigating Translation Competence: Conceptual and Methodological Issues". Meta 50:2. pp. 609-619.

PACTE. (2008) "First Results of Translation Competence Experiment: 'Knowledge of Translation' and 'Efficacy of the Translation Process"'. En: Kearns, John (ed.) 2008. Translator and Interpreter Training. Issues, Methods and Debates. Londres \& Nueva York: Continuum Studies in Translation. pp. 104-126.

Quivy, Raymond \& Luc van Campenhoudt. (2005) Manual de investigación en ciencias sociales. México: Limusa.

VAlERO, Oliver; Anna Espinal \& Pere Puig. (2008) "Analysis of labor spells in Social Security contributors". Proceedings of the 23rd International Workshop on Statistical Modelling. P.H.C Eilers editor, pp. 426 - 429.

VAlles, Miguel S. (1997) Técnicas cualitativas de investigación social. Madrid: Síntesis.

VAnde Walle, Jean Marie. (2006) "Evolutions de la profession - attentes, espoirs, perspectives". En: Gouadec, Daniel (dir.) 2006. Traduire pour le web. París: La Maison du Dictionnaire. pp. 81-85.

Verd, Joan M. \& Pedro López. (2008) "La eficiencia teórica y metodológica de los diseños multimétodo". EMPIRIA. Revista de Metodología de Ciencias Sociales 16. pp.13-42.

Widler, Brigitte. (2004) "A Survey Among Audience of Subtitled Films in Viennese Cinemas”. Meta 49:1. pp. 98-101. 